\title{
Predicción de la utilidad clínica de la radiografía de tórax rutinaria en una unidad de cuidados intensivos de traumatología
}

\author{
M. Chico Fernández*, A. Mohedano Gómez, C. García-Fuentes, P. Rico Cepeda, \\ A. Bueno González y E. Alted López
}

Unidad de Cuidados Intensivos de Traumatología y Emergencias, Hospital Universitario 12 de Octubre, Madrid, España

Recibido el 27 de julio de 2010; aceptado el 14 de marzo de 2011

\section{PALABRAS CLAVE \\ Cuidados críticos; Cuidados intensivos; Traumatismo grave; Unidad de cuidados intensivos ( $\mathrm{UCl}$ ); Radiografía de tórax}

\begin{abstract}
Resumen
Introducción: El valor de la radiografía torácica rutinaria está en cuestión. Sin embargo, este asunto no ha sido suficientemente evaluado en el caso concreto del paciente traumatizado. Nos propusimos encontrar predictores de utilidad en este entorno.

Material y métodos: Estudio observacional prospectivo en una $\mathrm{UCI}$ de traumatología de 8 camas. Se incluyeron los pacientes de más de 15 años, con traumatismo grave (ISS > 15), ingresados $48 \mathrm{~h}$ o más. Se excluyeron las mujeres embarazadas y las radiografías no rutinarias. Un miembro del equipo médico examinó de manera independiente las placas, en busca de hallazgos radiográficos según se definían en una lista cerrada. Durante la ronda diaria, los médicos al cargo comunicaron cuantos cambios en el estado clínico y en el manejo ocurrieron tras la toma de la radiografía, también siguiendo una lista de criterios cerrados. La relación entre ambas variables se estudió por análisis univariante y multivariante.

Resultados: Durante un año se obtuvieron 1.440 radiografías de 138 pacientes consecutivos. Predominaron los varones (82\%), de edad joven (39 \pm 1 años). El 97\% sufrieron traumatismo contuso; el 52\%, traumatismo torácico grave. La estancia media se prolongó 12,9 $\pm 10,1$ días. En el 86,8\% se empleó ventilación mecánica. De cada paciente se realizaron 10,4 $\pm 9,3$ placas. En el $14 \%$ hubo hallazgos relevantes, con mayor frecuencia una malposición de un dispositivo $(6,8 \%)$ o infiltrados $(4,9 \%)$, desencadenando una acción clínica en el $84,6 \%$ de los casos. Por análisis multivariante, se encontraron los siguientes factores de riesgo significativos $(p<0,05)$ para un hallazgo radiográfico: primeros 2 días de evolución, ventilación mecánica, empeoramiento de la $\mathrm{PaO}_{2} / \mathrm{FiO}_{2}$, empeoramiento de la distensibilidad pulmonar y variación en las secreciones respiratorias.

Conclusión: De acuerdo con estos datos, existe un riesgo pequeño de pasar por alto condiciones peligrosas si se restringe el uso de las radiografías rutinarias a las situaciones predictivas descritas. El observar esta política probablemente significaría un sustancial ahorro económico y de la emisión de radiaciones.

(c) 2010 Elsevier España, S.L. y SEMICYUC. Todos los derechos reservados.
\end{abstract}

\footnotetext{
* Autor para correspondencia.

Correo electrónico: murgchico@yahoo.es (M. Chico Fernández).
} 


\section{KEYWORDS}

Critical care;

Intensive care;

Severe trauma;

Intensive care unit

(ICU);

Chest radiograph
Prediction of the clinical usefulness of routine chest X-rays in a traumatology ICU

\begin{abstract}
Background: The clinical value of routine chest X-rays in critical care has been questioned, but has not been studied in the trauma environment to date. The objective of this study was to identify easy to use clinical predictors of utility in this setting.

Material and methods: A prospective observational study was made in an 8-bed traumatology ICU. Severe trauma patients (ISS > 15), aged 15 or older and admitted for $48 \mathrm{~h}$ or longer were included. Pregnant women and radiographs obtained during initial care or for reasons other than routine indication were excluded. A staff physician, separated from clinical duties, independently reviewed the films in search of changes, as described in a closed checklist. Following closed criteria, the attending physicians reported previous day clinical events and changes in clinical management after chest X-ray obtainment. Demographic and epidemiological data were also recorded. The associations among variables were studied by univariate and multivariate analysis.

Results: A total of 1440 routine chest X-rays were obtained from 138 consecutive patients during one year. Young males prevailed (82\%; $39 \pm 16$ years). The most common process was severe blunt trauma (97\%). Fifty-two percent suffered severe chest trauma. The mean length of stay was $12.9 \pm 10.1$ days. Mechanical ventilation was used in $86.8 \%$ of the cases. A median of $10.4 \pm 9.3$ films were obtained from each patient. A total of $14 \%$ of the X-rays showed changes, most commonly malpositioning of an indwelling device $(6.8 \%)$ or infiltrates $(4.9 \%)$. Those findings led to a change in care in $84.6 \%$ of the cases. Multivariate analysis identified the following significant $(p<0.05)$ risk factors for radiographic changes: first two days of evolution, mechanical ventilation, worsening of $\mathrm{PaO}_{2} / \mathrm{FiO}_{2}$, worsening of lung compliance and changes in respiratory secretions.

Conclusions: Based on the results obtained, the risk of not identifying dangerous conditions by restricting routine chest $X$-rays prescription to the described conditions is low. Observing this policy would probably mean substantial savings and a reduction in radiation exposure.

(c) 2010 Elsevier España, S.L. and SEMICYUC. All rights reserved.
\end{abstract}

\section{Introducción}

La radiografía torácica rutinaria $(R X R)$ es la que se obtiene del paciente crítico de una manera programada generalmente al comienzo de la jornada diaria- sin otra indicación clínica previa que el estar ingresado en una $\mathrm{UCI}$. Este uso puede asimilarse a las técnicas de cribado dirigidas a anticiparse a una condición potencialmente grave, como en el paciente crítico puedan ser neumotórax, atelectasias, derrames u otras ${ }^{1}$. Aunque es un recurso de empleo común, su validez es puesta en duda por un número creciente de autores. Tras los resultados de varios estudios recientes, algunos centros han eliminado su práctica. No obstante, no existían estudios sobre la materia en el ambiente de la UCI traumatológica.

Se han señalado numerosos peligros derivados de su uso (falta de calidad de los equipos de radiología portátiles que no garantizan una calidad de imagen adecuada en las condiciones del paciente crítico $^{2}$, generando la posibilidad del sobre o infradiagnóstico de enfermedades de importancia), el riesgo de la movilización de pacientes inestables, los costes $y$, por último, la exposición del paciente y el entorno a la radiación.

Atendiendo a estas consideraciones, se han llevado a cabo varios estudios, sin conclusiones unánimes. A pesar de ello, el panel de expertos de Colegio Americano de Radiología recomienda su uso en pacientes con enfermedades cardiopulmonares agudas o bajo ventilación mecánica ${ }^{3}$, tal como pueden estar la mayoría de los pacientes críticos en cualquier momento determinado.

Los enfoques de RXR frente solo a demanda no tienen por qué ser antagónicos, sino complementarios. Nuestro objetivo es encontrar factores predictores de utilidad de la RXR.

\section{Material y métodos}

Efectuamos un estudio observacional prospectivo, durante 12 meses, en la UCI de Traumatología y Emergencias, del Hospital Universitario 12 de Octubre. Este centro, de nivel terciario y titularidad pública, dispone de unas 1.400 camas ( 8 en esta unidad) y atiende un área de referencia sanitaria que cubre la zona sur de la ciudad de Madrid y su provincia con una cobertura de una población de 750.000 personas.

Cada mañana, se obtuvieron las RXR mediante un equipo de radiografía digital portátil. Se definió la RXR como la tomada por este medio, de manera programada, al inicio de la jornada (8 AM), sin que mediase ningún acontecimiento clínico que desencadenase la petición. Se incluyeron los pacientes mayores de 15 años, afectados de traumatismo grave (ISS > 15), ingresados durante $48 \mathrm{~h}$ - más. Se excluyeron las mujeres embarazadas y las radiografías de las primeras $24 \mathrm{~h}$ de ingreso o demandadas por motivos específicos. Así, se descartaron las radiografías indicadas por clínica o por realización de técnicas invasivas o manipulación de dispositivos internos (catéteres venosos 
Tabla 1 Hallazgo radiográfico nuevo, aparición o aumento significativo de un fenómeno en la imagen de la radiografía

\begin{tabular}{ll}
\hline Atelectasia & $\geq 1$ lóbulo o que aumente $\geq$ \\
Infiltrado & 1 lóbulo \\
& $\geq 1$ cuadrante o que \\
& aumente $\geq 1$ cuadrante \\
Derrame pleural & Ocupación de seno \\
& costofrénico, \\
& engrosamiento cisural, \\
& aumento de densidad \\
& homogéneo, difuminación \\
& de hemidiafragma o de \\
& vasculatura pulmonar \\
& inferior \\
Neumotórax o & Visualización de aire \\
neumomediastino & ectópico \\
Malposición de tubo traqueal & $<2$ cm de carina o por \\
& encima del espacio \\
& interclavicular \\
Malposición de dispositivos & Punta en aurícula derecha o \\
endovasculares & fuera de la luz o desplazada \\
Malposición de sondas & Punta de naso/orogástrica \\
digestivas & fuera del estómago \\
Malposición de drenaje & Desplazamiento $>5$ cm o \\
endotorácico & fuera del espacio pleural \\
\hline
\end{tabular}

centrales, catéter de arteria pulmonar, tubo orotraqueal, traqueostomía, toracocentesis, drenaje endotorácico, sondaje digestivo, broncoscopia, cirugía u otros).

Para cada variable (hallazgos radiológicos nuevos, cambios en la actitud clínica y en la situación clínica del paciente) de estudio se atendió a una definición estricta, tal como se muestra en las tablas $1-3^{4}$. Se recogieron datos clínico-epidemiológicos de los pacientes (tabla 4). Un médico de la plantilla, liberado de tareas de atención clínica y no implicado directamente en el estudio, revisó las RXR tomadas cada día, en busca de nuevos hallazgos radiológicos, según las definiciones preestablecidas. Al día siguiente, el equipo dedicado a la atención se encargaba de la revisión para detectar cambios secundarios en el manejo de cada paciente en las horas siguientes a la obtención de la radiografía. Como variables predictivas, se buscó la existencia de cambios en la evolución de cada paciente en el día previo a la radiografía, también según definiciones preestablecidas.

Los valores de cada variable se recogieron e introdujeron en una base de datos (Access; Microsoft, Redmond, Washington, USA) creada para tal fin.

Las variables categóricas se expresan como frecuencia relativa, en porcentajes; las variables cuantitativas como la media \pm desviación estándar. La comparación de variables

Tabla 2 Cambios en la actitud clínica: consumo de un recurso que genere una actitud sobre el paciente

Iniciación de cambios posturales

Obtención de muestras microbiológicas

Inicio de antibioterapia

Implantación o recolocación de dispositivos

Solicitud de técnica de imagen (eco/TAC/broncoscopia)
Tabla 3 Cambios en la situación clínica del paciente: eventos acaecidos en las $24 \mathrm{~h}$ previas a la radiografía

\begin{tabular}{|c|c|}
\hline Inestabilidad hemodinámica & $\begin{array}{l}\text { - TAS }<90 \mathrm{~mm} \text { de } \mathrm{Hg} \\
\text { - Requerimientos de fármacos } \\
\text { vasoactivas o incremento de } \\
\text { su dosis } \\
\text { - Infusión de volumen en bolo }\end{array}$ \\
\hline $\begin{array}{l}\text { Cambio en la distensibilidad } \\
\qquad\left(\mathrm{ml} / \mathrm{cmH}_{2} \mathrm{O}\right)\end{array}$ & $\geq 1$ grado LIS ${ }^{a}$ \\
\hline Cambio en la PEEP & $\geq 1$ grado según LIS ${ }^{a}$ \\
\hline $\begin{array}{l}\text { Cambio en la } \mathrm{PaO}_{2} / \mathrm{FiO}_{2} \\
\text { Colocación dispositivo } \\
\text { Desaturación }<90 \% \\
\text { SRIS }\end{array}$ & $\geq 1$ grado según LIS $^{a}$ \\
\hline $\begin{array}{l}\text { Cambio en las secreciones } \\
\text { respiratorias }\end{array}$ & Color/viscosidad/cantidad \\
\hline Ventilación mecánica & No/intermitente/sí \\
\hline
\end{tabular}

categóricas se realizó con el test de $\chi^{2}$ con un nivel de significación estadística de $p<0,05$. En el análisis multivariante se aplicó un modelo de regresión logística binaria en el que la variable dependiente es la aparición de hallazgos nuevos en la RXR de la mañana y donde las variables independientes se incluyen en función no solo la significación estadística del análisis univariante sino también la pertinencia, normalidad y colinealidad de las variables analizadas. Los resultados del análisis multivariante se expresan como riesgo relativo (RR) con su intervalo de confianza del 95\% (IC 95\%).

El análisis estadístico se llevó a cabo mediante SPSS, versión 15 (SPSS Inc.; Chicago, Illinois, USA).

\section{Resultados}

El estudio duró 12 meses entre marzo de 2006 y febrero de 2007. Al final de este período el estudio se detuvo al comprobar una significación suficiente para replantear nuestra política de prescripción de las RXR. Se seleccionaron 138 pacientes y se estudiaron 1.440 RXR $(10,4 \pm 9,4$ $\mathrm{RXR} /$ paciente). El promedio de estancia fue de $13 \pm 10$ días. El $81,9 \%$ de los pacientes fueron varones. La distribución de la gravedad del traumatismo torácico según el Maximum Abbreviated Injury Score (MAIS) fue: sin lesión (0) el $45,3 \%$, con lesión menor (1-2) el 2,8\% y con lesión grave (3-5) el $51,9 \%$. El resto de las variables demográficas y clínicoepidemiológicas se muestran en las tablas 4 y 5 .

Tabla 4 Variables demográficas

\begin{tabular}{ll}
\hline & $\begin{array}{l}\text { Porcentaje } \\
\text { Media } \pm \mathrm{DE}\end{array}$ \\
\hline Varones & $82 \%$ \\
Edad & $39,5 \pm 15,9$ \\
ISS & $33,4 \pm 12,6$ \\
Estancia & $13 \pm 10$ \\
Días de ventilación mecánica & $9 \pm 8$ \\
$\mathrm{~N} .{ }^{\circ}$ radiografías rutinarias & $10,4 \pm 9,4$ \\
$\mathrm{~N} .{ }^{\circ}$ radiografías no rutinarias & $2,7 \pm 3,9$ \\
\hline
\end{tabular}


Tabla 5 Hallazgos radiográficos

\begin{tabular}{ll}
\hline & Porcentaje \\
\hline Malposición dispositivos & 6,8 \\
- Tubo traqueal & 5 \\
- Sonda digestiva & 0,9 \\
- Catéter vascular & 0,6 \\
- Drenaje endotorácico & 0,3 \\
Otros hallazgos & 8,8 \\
- Condensación & 4,9 \\
- Atelectasia & 2,2 \\
- Derrame & 1,3 \\
- Neumotórax & 0,5 \\
- Lesión torácica oculta & 0,21 \\
\hline
\end{tabular}

Fueron pérdidas 8 RXR para el análisis debido a inconvenientes técnicos o de filiación. El $14 \%$ de las restantes mostraron nuevos hallazgos (más de uno en el $1,5 \%$ ). No se encontró ningún hallazgo radiográfico en el $86 \%$, un hallazgo en el $12,5 \%$ y 2 en el $1,5 \%$ de los casos. Los más frecuentes fueron la malposición de dispositivos $(6,8 \%)$ y los infiltrados pulmonares $(4,9 \%)$. En la tabla 6 se detallan las frecuencias de los restantes hallazgos.

Se encontró que en el 11,9\% del total de las RXR desencadenaron un cambio en el manejo, aquellas con nuevos hallazgos lo supusieron en el $84,6 \%$ de los casos. En la tabla 7 se muestra una explicación más detallada.

Los resultados analíticos se obtuvieron por el estudio de la relación entre las variables «cambio clínico en el día previo» $\mathrm{y}$ «hallazgos radiográficos» mediante regresión logística y uni-multivariante según se detalla en los métodos. De acuerdo con ello, se encontraron los siguientes factores predictivos de hallazgos radiográficos nuevos: RXR de los primeros 2 días de evolución, caída de la $\mathrm{PaO}_{2} / \mathrm{FiO}_{2}$, SatO $>90 \%$, ventilación mecánica, cambio en la distensibilidad pulmonar, variación en el aspecto de las secreciones respiratorias y la toma de radiografía no rutinaria. El análisis multivariante mostró como factores predictivos significativos $(p<0,05)$ los siguientes: RXR de los primeros 2 días de evolución (RR 1,7; IC 95\% 1,2-2,5), descenso de la $\mathrm{PaO}_{2} / \mathrm{FiO}_{2}$ (RR 3; IC 95\% 2-4,5), ventilación mecánica, (RR 2,1; IC 95\% $1,2-3,5)$, cambio en la distensibilidad pulmonar (RR 1,9; IC $95 \% 1,1-3,5)$, y variación en las secreciones respiratorias (RR 3,9; IC 95\% 2,4-6,5). Los resultados analíticos se muestran en la tabla 7.

\section{Discusión}

Que conozcamos, este es el único trabajo centrado en una unidad de críticos traumatológicos. Con él, hemos definido el perfil del paciente en que la radiografía torácica rutinaria es más rentable en términos de modificaciones de manejo clínico.

Como sus limitaciones cabe reconocer 2 puntos fundamentales, la ausencia de valoración de las radiografías por radiólogo independiente y el empleo de una metodología no comparativa. En cuanto a lo primero, cabe argumentar que en nuestro entorno sanitario no es común la disponibilidad de un radiólogo para la evaluación diaria de RXR, siendo tarea del clínico encargado de la atención. Esto puede contribuir a la asimilación del estudio con el entorno real de aplicación. De todos modos, intentamos minimizar el sesgo mediante la evaluación independiente de las Rx por otro clínico del equipo. En cuanto a la carencia de estrategia comparativa, es lo común de la mayoría de los estudios habidos hasta el momento. En todo caso, no parece aplicable en este estado del conocimiento, dada la escasez de datos sobre las distintas estrategias potenciales a comparar.

En nuestro estudio, el número de RXR con nuevos resultados es escaso (14\%), pero, sin embargo, estos hallazgos son útiles en el $84 \%$ de las ocasiones. Esto puede ser un argumento tanto a favor como en contra del uso de estas radiografías. La solución podría darla la búsqueda de las situaciones que predigan la aparición de cambios radiográficos, permitiendo restringir las peticiones a estos casos, sin perder rendimiento. Nosotros hallamos relación significativa con la presencia previa del descenso en la $\mathrm{PaO}_{2} / \mathrm{FiO}_{2}$, empleo de ventilación mecánica, cambio en la distensibilidad pulmonar, variaciones en el aspecto de las secreciones respiratorias y realización de las radiografías en los primeros 2 días del ingreso.

Un enfoque similar al nuestro fue llevado a cabo por Fong et $\mathrm{al}^{5}$ en 1995. Mediante análisis multivariante en una cohorte de incepción, concluyen que la única justificación de la RXR en UCl es la presencia de un catéter de arteria pulmonar. Difiere en haber sido llevado a cabo en una $\mathrm{UCI}$ quirúrgica y en emplear definiciones que no coinciden con las del nuestro. Sin embargo, la proporción de hallazgos (26\%) y su rendimiento en el $65 \%$ son parecidos.

Los estudios prospectivos realizados hasta el momento en general han considerado importante la proporción de resultados radiológicos (35\% de media), aunque menos frecuentes que en las radiografías no rutinarias. No obstante, cuando se considera la utilidad, la mayoría son de poca envergadura y no promueven cambios. En el presente trabajo, el rendimiento solo alcanza el $14 \%$. Esto está lejos del 35\% de la mayoría de publicaciones pero próximo al de trabajos más recientes como el Graat et $\mathrm{al}^{6}(6,6 \%)$, de método y definiciones semejantes. Nuestra proporción de modificaciones de actitud es del $12 \%$, entre estudios más antiguos (20\%) y el de Graat et al $(2,2 \%)$. Si nos restringimos a las radiografías con hallazgos, esta proporción alcanza un $84 \%$. En los restantes estudios $^{1-3,7-9}$ hemos calculado un 37\%; Graat et $\mathrm{al}^{6}$

Tabla 6 Relación entre hallazgos radiográficos y cambios clínicos

\begin{tabular}{llll}
\hline & Con cambio de actitud & Sin cambio de actitud & Total \\
\hline Radiografía con hallazgos & $170 / 201(84,6 \%)$ & $31 / 201(15,4 \%)$ & $201 / 1432(14 \%)$ \\
Radiografía sin hallazgos & $0 / 1.231(0 \%)$ & $1.231 / 1.231(100 \%)$ & $1.239 / 1.432(86 \%)$ \\
Total & $170 / 1.432(11,9 \%)$ & $1.262 / 1.432(88,1 \%)$ & 1.432 \\
\hline
\end{tabular}


Tabla 7 Análisis univariante y multivariante

\begin{tabular}{llll}
\hline Evento clínico & Análisis univariante & \multicolumn{2}{c}{ Análisis multivariante } \\
\cline { 3 - 4 } & & RR $(I C 95 \%)$ & Significación estadística \\
\hline$\leq 2$ días evolución vs $\geq 3$ & $\mathrm{p}<0,00$ & $1,9(1,2-2,5)$ & $\mathrm{p}=0,007$ \\
Disminución $\mathrm{PaO}_{2} / \mathrm{FiO}_{2}$ & $\mathrm{p}<0,00$ & $3,02(2,01-4,56)$ & $\mathrm{p}=0,000$ \\
Ventilación mecánica & $\mathrm{p}<0,00$ & $2,1(1,26-3,51)$ & $\mathrm{p}=0,004$ \\
Disminución de distensibilidad & $\mathrm{p}<0,00$ & $1,9(1,03-3,83)$ & $\mathrm{p}=0,038$ \\
Inestabilidad hemodinámica & $\mathrm{p}=0,003$ & $0,87(0,47-1,60)$ & $\mathrm{p}=0,663$ \\
SRIS & $\mathrm{p}=0,001$ & $1,23(0,70-2,16)$ & $\mathrm{p}=0,468$ \\
Cambio en secreciones & $\mathrm{p}<0,00$ & $3,98(2,42-6,54)$ & $\mathrm{p}=0,000$ \\
Cambio en dispositivos & $\mathrm{p}=0,727$ & $0,69(0,38-1,25)$ & $\mathrm{p}=0,229$ \\
Radiografía no rutinaria & $\mathrm{p}<0,00$ & $1,35(0,85-2,15)$ & $\mathrm{p}=0,199$ \\
\hline
\end{tabular}

alcanzan el 32\%, y Fong et $\mathrm{al}^{5}$ el $65 \%$. Estas diferencias pueden deberse a distintos métodos y definiciones, lo que dificulta las comparaciones.

Puede resultar llamativa la baja proporción de lesiones traumáticas ocultas que son halladas por vez primera mediante RXR en este estudio $(0,21 \%)$. La anticipación de diagnósticos por evaluación terciaria ${ }^{10}$ puede explicar tan bajo rendimiento en este aspecto.

La mayoría de los estudios anteriores se han realizado en UVI médicas, quirúrgicas o multidisciplinarias, lo que explica que en la población de nuestro estudio (de traumatología) predominen los varones (82 frente a 60\%) y la edad media sea menor (39 frente a 65 años). El tamaño de la muestra, de la unidad de estudio, la duración del seguimiento y el número de radiografías recogidas están en valores intermedios a lo mostrado por publicaciones previas (3 a 28 camas, 80 a 754 pacientes, 220 a 2.475 radiografías y 4 a 25 meses) $)^{1,3,5,6,8,9,11,12}$.

Una de las principales opiniones en contra de la práctica de las RXR se deriva de la preocupación acerca de la radiación recibida por el paciente y el espacio circundante. A este respecto, cabe argüir que algunas investigaciones ${ }^{3,13-15}$ muestran que la radiación ambiental acumulada está por debajo del nivel de riesgo con las pautas de uso convencionales.

Sin embargo, la más poderosa oposición procede de la percepción de los costes económicos resultantes. En estudios de los años 1990 cada radiografía suponía a un gasto de unos 75 \$ que ascendían hasta a 120 \$ para el pagador final. A este respecto, los trabajos son heterogéneos y difíciles de comparar, con resultados incluso opuestos. Price et $\mathrm{al}^{16}$, en1999, encontraron un importante ahorro con la política restrictiva en una $\mathrm{UCl}$ pediátrica en la que se hicieron 4.550 radiografías rutinarias durante 30 meses. Otros hallan ahorros que se derivarían de la anticipación de diagnósticos, como, por ejemplo, Brainsky et $\mathrm{al}^{8}$ (1997), con ahorro de $98 \$$ por radiografía tomada. $\mathrm{El}$ empeño actual en la reducción de gastos y la racionalización de recursos en la práctica sanitaria puede terminar por limitar la prescripción de pruebas complementarias a los supuestos admitidos en protocolos cerrados ${ }^{17}$. La utilidad de las radiografías de control tras la colocación de dispositivos internos está también en duda. Posiblemente, aquellas instrumentaciones practicadas en enfermos adecuadamente monitorizados, puedan diferirse o restringirse a los casos de más precaución, con ahorro de hasta 150 \$ por placa $^{18-22}$.

Establecer un umbral de utilidad es difícil partiendo de estudios observacionales, sea cual sea el parámetro elegido como comprobación. La respuesta deberá venir de estudios comparativos que enfrenten las distintas estrategias en resultados de peso como morbilidad y mortalidad, estancia o costes. Kripoval et al $^{12}$ (2003) compararon la estrategia rutinaria con la restrictiva en una investigación prospectiva aleatorizada en una UCI médica de 20 camas. Durante 10 meses se dirige a los enfermos al grupo de radiografías rutinarias o al grupo que las recibe solamente por indicación específica. El segundo recibió menos radiografías y no hubo diferencias en la duración de la ventilación mecánica, la estancia ni la mortalidad. Con este descenso del número de radiografías, se asumió también una reducción de los costes y de la exposición a radiación. En su contra está el ser un estudio pequeño (94 pacientes), que no incluye situaciones complejas que podrían ser las más beneficiadas por las RXR (ventilación prolongada o en prono, casos trasladados, etc.), lo que dificulta la generalización de sus conclusiones.

Diversos autores han analizado la utilidad de las radiografías rutinarias demostrando la escasa utilidad de las mismas ${ }^{23,24}$ y que tras su retirada no han encontrado cambios en la morbilidad y mortalidad de sus pacientes; no obstante, dado que no se trata de estudios aleatorizados, sus resultados deben tomarse con cautela. Considerando todos los elementos previos, parecería necesario plantearse individualizar la realización de RXR. Pero, por el momento, no es seguro concluir que esta sea una actitud adaptable, segura y aplicable en todos los entornos hasta que esto sea comprobado en estudios de mayor calidad. Recientemente, Mets et $\mathrm{al}^{25}$, en una unidad cardiotorácica, y Hendriske et $\mathrm{al}^{26}$, en una UCI mixta médico-quirúrgica, han ayudado a ir aclarando las ideas. Tras un cambio en la política radiológica, en la que eliminan las RXR, comparan los resultados de ambas fases, obteniendo buenos resultados en mortalidad, estancia y tasa de reingresos.

\section{Conclusión}

El riesgo de pasar por alto condiciones peligrosas al restringir el uso de las radiografías rutinarias parece bajo como 
contrapartida, podrá suponer un significativo ahorro económico y en la emisión de radiaciones. No obstante, se requieren nuevos estudios que confirmen estos resultados en diversos tipos de pacientes.

\section{Conflicto de intereses}

Los autores declaran no tener ningún conflicto de intereses.

\section{Bibliografía}

1. Bekemeyer WB, Crapo RO, Calhoon S, Clayton PD. Efficacy of chest radiography in a respiratory intensive care unit. A prospective study. Chest. 1985;88:691-6.

2. Savoca CJ, Gamsu G, Rohlfing BM. Chest radiography in intensive care units. West J Med. 1978;129:469-74.

3. Graat ME, Stoker J, Vroom MB, Schultz MJ. Can we abandon daily routine chest radiography in intensive care patients? J Intensive Care Med. 2005;20:238-46.

4. Murray JF, Matthay MA, Luce JM, Flick MR. A expanded definition of the adult respiratory distress syndrome. Am Rev Respir Dis. 1988;138:720-3.

5. Fong Y, Whalen GF, Hariri RJ, Barie PS. Utility of routine chest radiographs in the surgical intensive care unit: a prospective study. Arch Surg. 1995;130:764-8.

6. Graat ME, Choi G, Wolthuis EK, Korevaar JC, Spronk PE, Stoker J, et al. The clinical value of daily routine chest radiographs in a mixed medical-surgical intensive care unit is low. Crit Care. 2006;10:R11.

7. Hall JB, White SR, Karrison T. Efficacy of daily routine chest radiographs in intubated, mechanically ventilated patients. Crit Care Med. 1991;19:689-93.

8. Brainsky A, Fletcher RH, Glick HA, Lanken PN, Williams SV, Kundel HL. Routine portable chest radiographs in the medical intensive care unit: Effects and costs. Crit Care Med. 1997;25:801-5.

9. Chahine-Malus N, Stewart T, Lapinsky SE, Marras T, Dancey D, Leung $R$, et al. Utility of routine chest radiographs in a medicalsurgical intensive care unit: a quality assurance survey. Crit Care. 2001;5:271-5.

10. Biffl WL, Harrington DT, Cioffi WG. Implementation of a tertiary trauma survey decreases missed injuries. J Trauma. 2003;54:38-44.

11. Graat ME, Kroner A, Spronk PE, Korevaar JC, Stoker J, Vroom $M B$, et al. Elimination of daily routine chest radiographs in a mixed medical-surgical intensive care unit. Intens Care Med. 2007;33:639-44.

12. Krivopal M, Shlobin OA, Schwartzstein RM. Utility of daily routine portable chest radiographs in mechanically ventilated patients in the medical ICU. Chest. 2003;123:1607-14.
13. Weatherburn GC, Bryan S, Davies JG. Comparison of doses for bedside examinations of the chest with conventional screen-film and computed radiography: results of a randomized controlled trial. Radiology. 2000;217:707-12.

14. Pandit-Bhalla M, Diethelm L, Espenan G. Portable chest radiographs in the intensive care units: referral patterns and estimated cumulative radiation exposures. J Thorac Imaging. 2002;17:211-3.

15. Mostafa G, Sing RF, McKeown R, Huynh TT, Heniford BTI. The hazard of scattered radiation in a trauma intensive care unit. Crit Care Med. 2002;30:574-6.

16. Price MB, Grant MJC, Welkie K. Financial impact of elimination of routine chest radiographs in a pediatric intensive care unit. Crit Care Med. 1999;27:1588-93.

17. Wang TJ, Mort EA, Nordberg P, Chang Y, Cadigan ME, Mylott L, et al. A utilization management intervention to reduce unnecessary testing in the coronary care unit. Arch Intern Med. 2002;162:1885-90.

18. Brunel W, Coleman DL, Schwartz DE, Peper E, Cohen NH. Assessment of routine chest roentgenograms and the physical examination to confirm endotracheal tube position. Chest. 1989;96:1043-5.

19. Amshel CE, Palesty JA, Dudrick SJ. Are chest x-rays mandatory following central venous recatheterization over a wire? Am Surg. 1998;64:499.

20. Frassinelli P, Pasquale MD, Cipolle MD, Rhodes M. Utility of chest radiographs after guidewire exchanges of central venous catheters. Crit Care Med. 1998;26:611-5.

21. Lotano R, Gerber D, Aseron C, Santarelli R, Pratter M. Utility of postintubation chest radiographs in the intensive care unit. Crit Care. 2000;4:50-3.

22. Pizano LR, Houghton DE, Cohn SM, Frish MS, Grogan RH. When should a chest radiograph be obtained after chest tube removal in mechanically ventilated patients? A prospective study. J Trauma. 2002;53:1073-7.

23. Tolsma $M$, Kroner $A$, van den Hombergh $C L$, Rosseel $P M$, Dijpstra TA, Bentala $M$, et al. The clinical value of routine chest radiographs in the first 24 hours after cardiac surgery. Anesth Analg. 2011;112:139-42.

24. García- Córdoba F, García Santos B, González Díaz G, García-Gerónimo A, Muñoz Zambudio F, et al. Decrease of unnecessary chest $\mathrm{x}$-rays in Intensive Care Unit: application of a combined cycle of quality improvement. Med Intens. 2008;3:71-7.

25. Mets O, Spronk PE, Binnekade J, Stoker J, de Mol BA, Schultz MJ. Elimination of daily routine chest radiographs does not change on-demand radiography practice in postcardiothoracic surgery patients. J Thorac Cardiovasc Surg. 2007;134:139-44.

26. Hendriske KA, Gratama JW, Hove W, Rommes JH, Schultz MJ, Spronk PE. Low value of routine chest radiographs in a mixed medical-surgical ICU. Chest. 2007;132:823-8. 